\title{
ANATOMICAL VARIATIONS AFFECTING THE SURGERY OF DE QUERVAIN'S DISEASE
}

\author{
K. W. GILES, LONDON, ENGLAND \\ From the Anatomy Department, the London Hospital Medical College
}

Operations designed to relieve the symptoms in stenosing tendovaginitis of the abductor pollicis longus and extensor pollicis brevis muscles (de Quervain's disease) involve surgical decompression of the osseo-fibrous canal in which the tendons lie. This canal, the first of the extensor tendon compartments at the wrist, is usually said to contain both these tendons in their respective synovial sheaths. Failure in this operation is common and may be related to anatomical variations in these two tendons. Such variations have been known for many years, Quain (1892) observing that the tendon of the abductor pollicis longus was the commonest of all the extensor tendons to vary, and Tillaux (1882) noting that the tendons of the abductor pollicis longus and extensor pollicis brevis might be found lying in separate fibrous compartments.

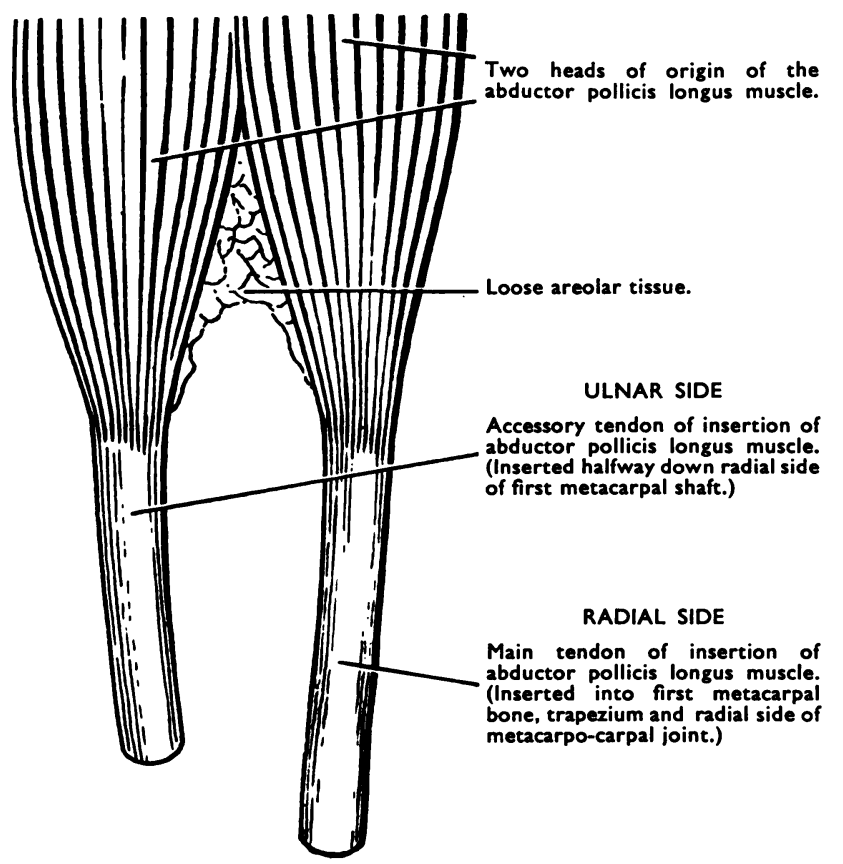

Fig. 1

Diagram of dissection of right abductor pollicis longus muscle of chimpanzee.

A study of the literature indicates that the standard anatomical description is the exception rather than the rule, and that the splitting of the abductor pollicis longus tendon into two or more lamellae is commonly found on dissection of the first extensor compartment of the wrist. Stein (1951) found in a series of eighty-four wrists that 68 per cent exhibited an accessory tendon of the abductor pollicis longus whereas only 4 per cent exhibited a similar accessory tendon of the extensor pollicis brevis. Baba (1954) in a study of 134 dissected wrists found that 132 (98.5 per cent) of these exhibited accessory tendons of the abductor pollicis longus, and, of these, seven showed the accessory tendon to be split into two or more lamellae.

The most important surgical aspect of these variations, normal rather than abnormal-is the incidence of separate osseo-fibrous compartments for these two main tendons or for their accessory tendons, and on this point the literature is scanty. Keon-Cohen (1951) observed in a series of sixty-six dissected forearms that 33 per cent showed the extensor pollicis brevis tendon to occupy a separate compartment. Therefore, in the surgery of stenosing tendovaginitis proper recognition of the presence of these accessory tendons and in particular the presence or absence of a separate osseo-fibrous compartment is of great importance. Decompression of the main osseo-fibrous canal may not relieve the symptoms of the disease if an accessory tendon remains unrecognised and is left compressed in its own fibrous canal. 
TABLE I

TABLE OF Findings

\begin{tabular}{|l|l|l|}
\hline Variations in the anatomy of the tendons involved in de Quervain's disease \\
\hline Accessory tendons of the abductor pollicis longus
\end{tabular}

Phylogenetically the abductor pollicis longus and extensor pollicis brevis are differentiations of a common muscle. This muscle appears infantile in this respect because its separation from the abductor pollicis longus is complete only in man and gorilla and the extensor pollicis brevis tendon is always much smaller than that of the abductor pollicis longus. Ayer (1948) described the abductor pollicis longus of the old world monkey as having insertions into the base of the thumb metacarpal bone, the trapezium and the flexor retinaculum [transverse carpal ligament], in this way resembling man. In one specimen he found a small prolongation from the main tendon of insertion, which extended down to the base of the proximal phalanx, such a prolongation probably representing the extensor pollicis brevis muscle. In a recent dissection of the chimpanzee (Pan) an accessory tendon of insertion (Fig. 1) of the abductor pollicis longus was found with an attachment half way along the radial side of the shaft of the thumb metacarpal bone. In the gorilla the abductor pollicis longus tendon divides one centimetre before its insertion into two nearly equal parts. The larger part is inserted into the trapezium and the rest is inserted into the lateral side of the base of the thumb metacarpal bone. The extensor pollicis brevis tendon has a double insertion; though mainly into the lateral aspect of the first metacarpal bone, it is also attached by a very slender tendon to the dorsal surface of the base of the proximal phalanx. Thus one notes a gradation in the extent of differentiation of this common muscle in different species. With such a process still in its phylogenetic infancy it is not surprising that anomalies are to be found in man with great relevance to the surgery of de Quervain's disease.

In this series a study was made of fifty dissected wrists, and particular note was made of the origin of the accessory tendon and of the presence or absence of a secondary osseo-fibrous canal within the first extensor compartment (Table I). An accessory tendon of the abductor pollicis longus was found in 56 per cent of wrists, though in only two wrists was the accessory tendon to be found lying in a separate fibrous canal. In the remainder the abductor pollicis longus with all its accessory tendons of insertion occupied a common canal, which was either the first extensor compartment or the anterior subdivision of this compartment in cases in which an accessory septum separated the extensor pollicis brevis and abductor pollicis longus tendons. The latter type of division of the main compartment into two parts, one for the extensor pollicis brevis and its accessory tendons, the other for the abductor pollicis longus and its accessory tendons, was found in 20 per cent of wrists. Such a division was easily recognised, though a limited surgical incision might have misled one into mistaking the anterior

vol. 42 B, NO. 2, MAY 1960 
compartment containing the abductor pollicis longus and its accessory tendon to be the whole of the first extensor compartment and its contents (Fig. 2).

The most interesting type of subdivision of the first extensor compartment affected the

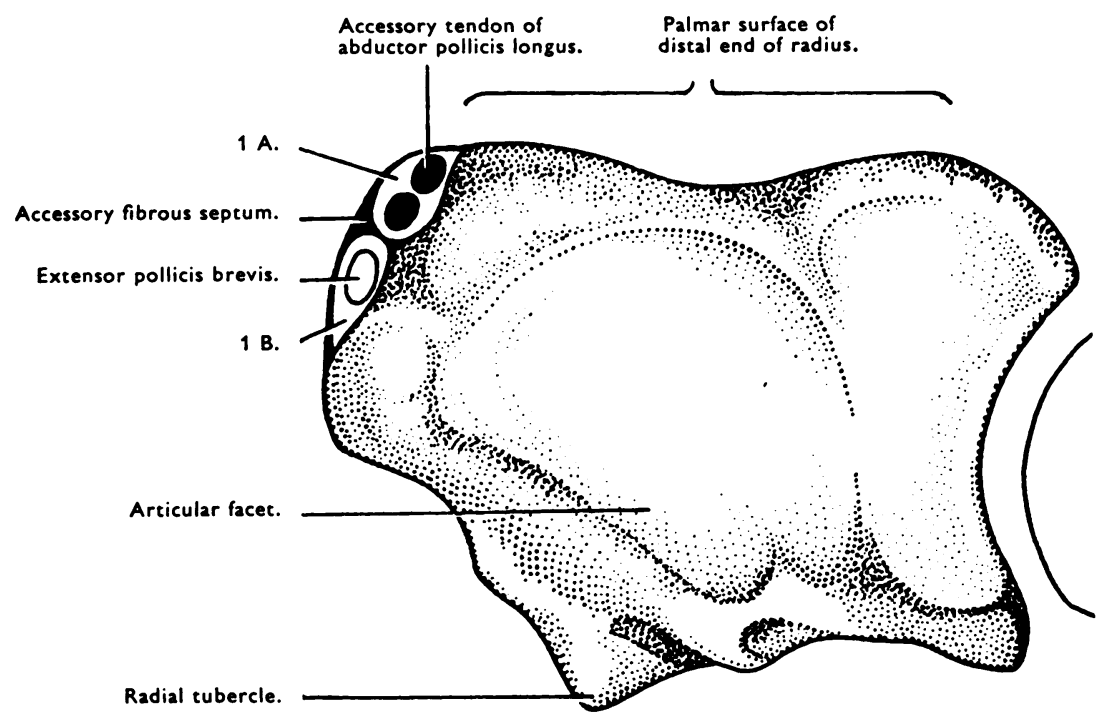

Fig. 2

Distal view of right radius (seen end on). 1A-Subdivision of first extensor compartment containing abductor pollicis longus plus accessory tendon. 1B-Subdivision of compartment containing extensor pollicis brevis.

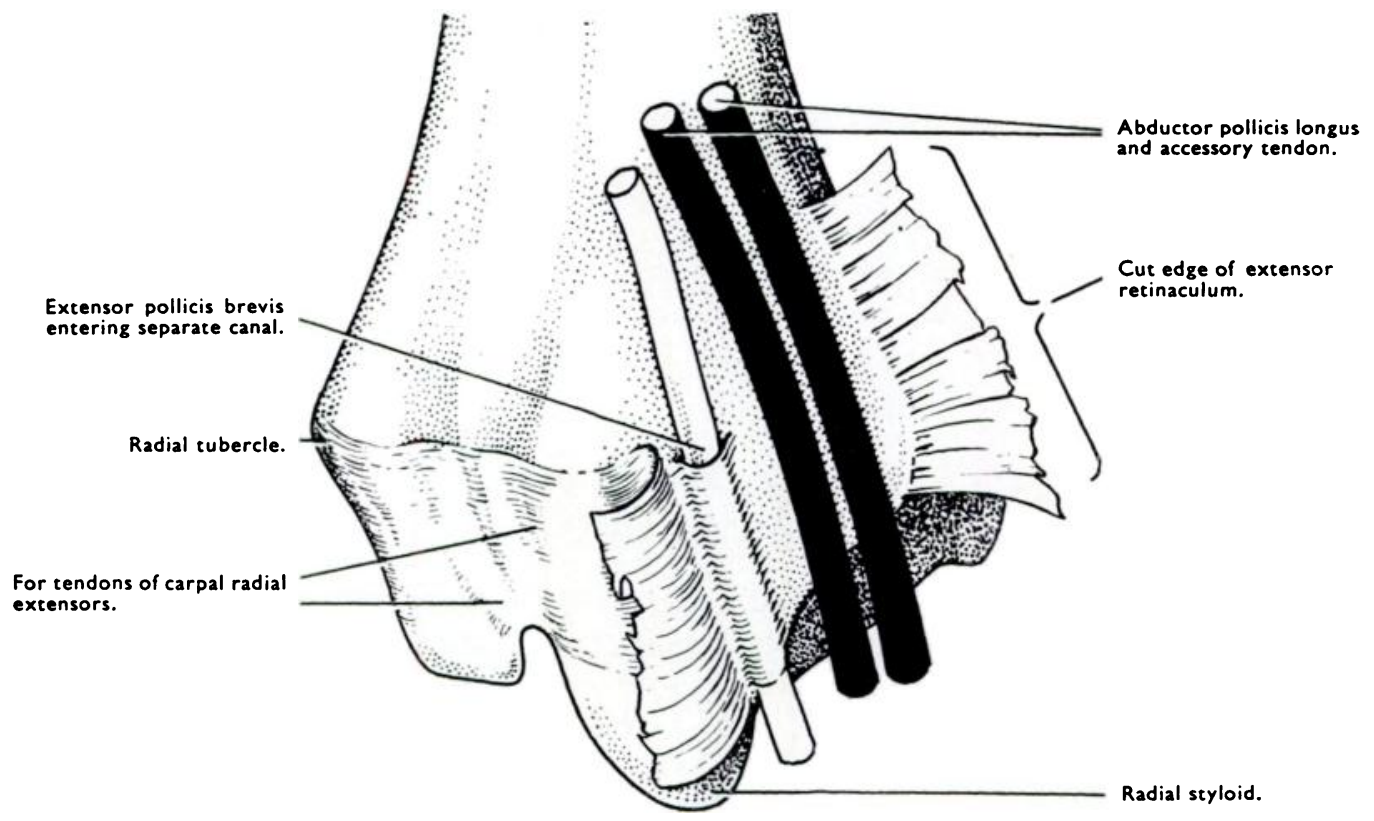

Fig. 3

Lateral view of distal end of right radius.

tendon of the extensor pollicis brevis. In 34 per cent of cases this tendon was found entering a separate canal, present only in the distal part of the compartment, often only the distal one-quarter. This separate osseo-fibrous canal lay deep and was found only after displacement 
of the overlying tendon or tendons of the abductor pollicis longus (Fig. 3). In each case the canal was small and the lumen seemed only just large enough to accommodate the tendon. Such circumstances, with a small and separate fibrous canal housing the extensor pollicis brevis tendon, would be aggravated in a corresponding stenosing tendovaginitis: furthermore, such a canal could well pass unobserved during a routine decompression of the first extensor compartment.

\section{SUMMARY AND CONCLUSIONS}

Attention has been drawn to the variations found in the anatomy of the tendons of the abductor pollicis longus and extensor pollicis brevis muscles as they lie in the first extensor compartment of the wrist. Such variations involve reduplication of the abductor pollicis longus tendon and more rarely the extensor pollicis brevis tendon. An accessory tendon may occasionally lie in a separate osseo-fibrous canal. It is not unusual to find the abductor pollicis longus and extensor pollicis brevis tendons lying in separate compartments, because an accessory fibrous septum is subdividing the first extensor compartment into two parts. Furthermore, the extensor pollicis brevis tendon may also lie in its own separate fibrous canal in the depths and the more distal part of this common compartment.

Such variation could lead to two possible misinterpretations during the course of surgical decompression for de Quervain's disease and these misinterpretations could reasonably be linked with the failure rate for this particular operation.

1. The finding of the abductor pollicis longus and its accessory tendon in a single compartment (12 per cent of wrists in the series) after a limited surgical incision could be mistaken for the abductor pollicis longus and extensor pollicis brevis tendons. Such a mistake would lead to failure to decompress the extensor pollicis brevis.

2. A failure to identify the deeper-lying separate canal for the extensor pollicis brevis in the distal part of the compartment would again be responsible for failure to decompress the extensor pollicis brevis (14 per cent of wrists in this series).

\section{REFERENCES}

Ayer, A. A. (1948): The Anatomy of Semnopithecus entellus. Madras: The Indian Publishing House Ltd.

BabA, M. A. (1954): The Accessory Tendon of the Abductor Pollicis Longus Muscle. Anatomical Record, $119,541$.

Gregory, W. K., ed. (1950): Anatomy of the Gorilla. New York: Columbia University Press.

KeON-COHEN, B. (1951): De Quervain's Disease. Journal of Bone and Joint Surgery, 33-B, 96.

LACEY, II, T., Goldstein, L. A., and Tobin, C. E. (1951): Anatomical and Clinical Study of the Variations in the Insertions of the Abductor Pollicis Longus Tendon, Associated with Stenosing Tendovaginitis. Journal of Bone and Joint Surgery, 33-A, 347.

Loomis, L. K. (1951): Variations of Stenosing Tenosynovitis at the Radial Styloid Process. Journal of Bone and Joint Surgery, 33-A, 340.

Parsons, F. G., and Robinson, A. (1899): Eighth Report of the Committee of Collective Investigation of the Anatomical Society of Great Britain and Ireland, for the Year 1897-98. Journal of Anatomy and Physiology, 33, 189.

Piersol, G. A. (1907): Human Anatomy. Philadelphia and London: J. B. Lippincott Company.

QuaIN, J. (1892): Elements of Anatomy. Tenth edition. Edited by E. A. Schäfer and G. D. Thane. Vol. 2, part 2. London: Longmans, Green, and Co.

Stein, A. H., Jun. (1951): Variations of the Tendons of Insertion of the Abductor Pollicis Longus and the Extensor Pollicis Brevis. Anatomical Record, 110, 49.

Tillaux, P. J. (1882): Traité d'anatomie topographique. Troisième édition. Paris: Asselin et Cie. 